\title{
Probing the Small Scale Structures around Herbig Be Stars using $\mathbf{H} \alpha$ Spectropolarimetry
}

\author{
René D. Oudmaijer, Janet E. Drew and Graeme Busfield
}

Astrophysics Group, Imperial College, London, U.K.

\begin{abstract}
.
We present spectropolarimetric observations across $\mathrm{H} \alpha$ of a sample of Herbig Be objects. Roughly half of the observed Herbig Be stars show polarization changes across $\mathrm{H} \alpha$, implying immediately that their ionized, hence inner, envelopes are not spherically symmetric. This pattern, if confirmed by observations of a larger sample, could indicate that the non-detection rate is simply a consequence of sampling randomly-oriented circumstellar disks able to scatter starlight within a few stellar radii, with as implication that the massive Herbig Be stars are still surrounded by accretion disks. In addition, we present preliminary modelling which will assist interpreting the kinematic structure of the electron-scattering region.
\end{abstract}

\section{Introduction}

A major obstacle to the understanding of the formation of massive stars is that we still do not know with certainty the geometry of their circumstellar material. From a theoretical perspective it is commonly accepted that during gravitational collapse, angular momentum conservation results in a circumstellar disk around a newly born star. This has been confirmed observationally for young, pre-main sequence analogues of the Sun, the so-called T Tau stars, around which potentially proto-planetary disks have been found. On the other hand, a theoretical consensus has not yet been reached for young higher mass stars, the Herbig Ae/Be stars. This problem stems mainly from the fact that theoretical work has so far failed to form massive objects the same way as lower mass stars, because radiation pressure may be capable of blowing away the accreting material, preventing the growth of stellar cores beyond $\approx 10 \mathrm{M}_{\odot}$. (e.g. Bonnell et al. 1998). Evidence that radiation pressure plays some role at higher masses is provided by Fuente et al. (1998, see also Böhm these proceedings) who found that Herbig Be stars have less sub-mm dust emission than A-type Herbig stars, suggestive of dispersal of the circumstellar dust by the stellar wind. Yet, the geometry of the accreting material is an important factor in determining how far the star growth is limited by radiation pressure since disk-like structures can provide shielding unavailable within spherical infall. Here is a good example of where theory needs input from observations to answer the fundamental question as to what the geometry is. However, from an observational perspective, a lively debate has developed over the last decade as to whether the circumstellar 

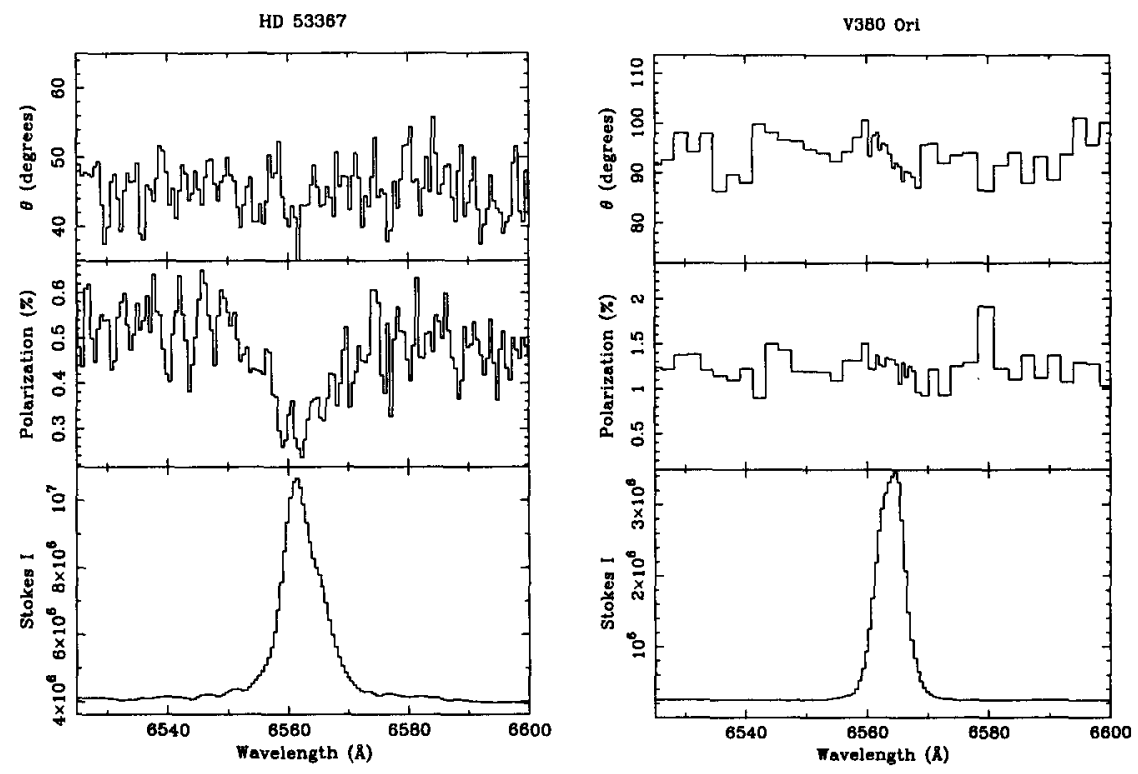

Figure 1. Example of a detection and a non-detection of the lineeffect in HD 53367 and V380 Ori respectively. The spectra have been re-binned to equal errors in polarization of $0.1 \%$. The lower panel shows the spectrum, while the middle and upper panels show the observed polarization and position angle respectively.

material of the Herbig $\mathrm{Ae} / \mathrm{Be}$ stars is disk-like or spherically symmetric (see Pezzuto et al. 1997 for a recent summary of the conflicting views). Unambiguous evidence that at least some of these objects have geometries deviating from spherical symmetry has only just appeared. Mannings et al. (1997) presented a direct detection in the millimeter regime of a rotating disk around the A-type Herbig star HD 31648.

\section{Spectropolarimetry around $\mathrm{H} \alpha$}

A powerful tool for discovering whether the ionized (hence inner) region around a star is flattened or not, is spectropolarimetry around the $\mathrm{H} \alpha$ line. When the observed polarization across the line differs from that of the neighbouring continuum (the 'line-effect'), one immediately knows that the electron-scattering region is not spherically symmetric. This is because the emission lines, forming in a larger volume, undergo less electron-scattering than the stellar continuum, and will thus be less polarized. With no effect across the line, the material is either spherically symmetric, located in a circular face-on disk, or there is a central hole in the disk such as suggested for $\mathrm{T}$ Tau stars. Electron scattering typically occurs at scales smaller than a few stellar radii (Cassinelli et al. 1987), far beyond the direct imaging capabilities presently available. The presence of the line-effect, therefore, is direct evidence of non-spherically symmetric structure 

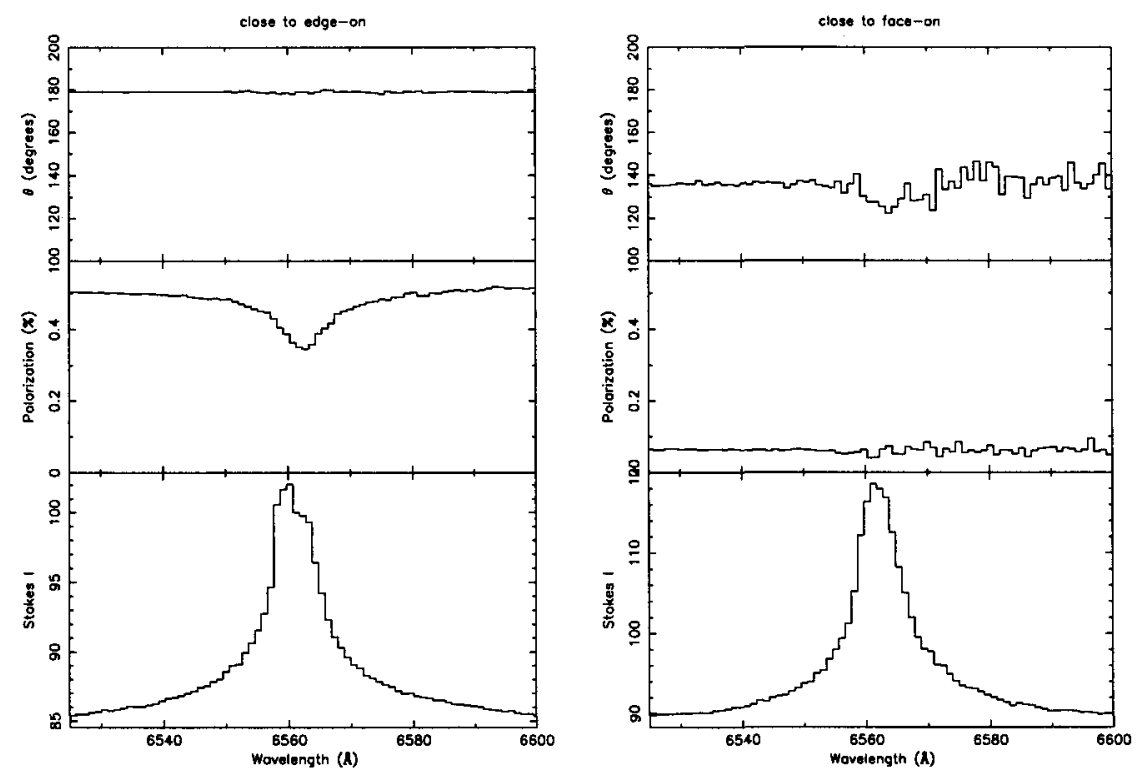

Figure 2. This plot shows a typical model of an outflowing source seen at different inclinations. Both the observed polarization and the 'line-effect' are less for the face-one case.

on small scales. Using this tool we have already shown in the case of the $\mathrm{B}[\mathrm{e}]$ star, HD 87643, that there is evidence of a rotating and expanding outflowing disk (Oudmaijer et al. 1998). Probably because of observational difficulties in the past, few studies such as these have been published in the literature (e.g. Schulte-Ladbeck et al. 1994, Harries et al. 1998), but none of these were targeted at young stars. Here we show the results of our pilot study on a sample of Herbig Be stars.

\section{Our pilot study}

We embarked upon a spectropolarimetric study of Herbig Be stars, using the RGO spectrograph with polarization optics mounted on the $3.9 \mathrm{~m}$ AAT, Australia. The set-up provided $60 \mathrm{~km} / \mathrm{s}$ resolution, while count rates in excess of 500000 per resolution element were required. One roughly needs to detect 1 million photons per resolution element to achieve an accuracy of $0.1 \%$ in polarization as the fractional error goes at $\sim 1 / \sqrt{N}$ ). The external accuracy however is limited to $0.1 \%$.

In total we observed 15 target objects from the catalogue by The et al. (1994), a list which inevitably is contaminated by other types of objects such as the evolved $\mathrm{B}[\mathrm{e}]$ stars (see Zickgraf, these proceedings). Indeed, a study by Oudmaijer et al. (1998) indicated that HD 87643 for example is more likely a luminous, evolved, B[e] star rather than a young Herbig Be object. Conservatively, 9 targets in our sample can be considered a Herbig Be star, and 4 of 
these, around half, show the line-effect (see Oudmaijer and Drew 1999). Figure 1 shows an example of an object with and one without the 'line-effect'.

The presence of disk-like structures around these stars allows that at least some of them are still surrounded by accretion-disks. But, as yet, the observed sample is not large enough to make a statistical assessment of the detectionrate. If this pattern continues to show after a larger sample has been observed, it is not impossible that almost all Herbig Be stars are surrounded by disks. This is because random inclinations will result in both detections and nondetections: a face-on disk does not show the line-effect as the scattering region is then circularly symmetric (see next section and fig. 2). Future observations are needed to test this.

\section{Prospects for modelling}

The power of this type of observations is that a detection of the line-effect provides an immediate answer to the qualitative question whether the electronscattering region is spherically symmetric. However, data such as these carry much more information. The velocity resolved structures can provide insights into the kinematics of the line-forming and scattering regions, Hence numerical modelling of the line-profiles and their resulting polarization will assist in the interpretation of the observed polarization spectra. We have begun such modelling, and have adopted Hillier's code (Hillier, 1996) to investigate the polarimetry of emission lines formed within ionized disks. Preliminary results, illustrating the inclination dependence of the line-effect are shown in Figure 2.

It is expected that this type of modelling, complementary to the broad-band polarization work by Bjorkman (these proceedings) will ultimately help to constrain the physical parameters of the disks, providing quantitative information to feedback into theoretical work on the formation of massive stars.

\section{References}

Böhm, T. these proceedings

Bjorkman, $\mathrm{K}$. these proceedings

Bonnell, I.A., Bate, M.R., Zinnecker, H. 1998, MNRAS 298, 93

Cassinelli, J.P., Nordsieck, K.H., Murison, M.A. 1987, ApJ 317, 290

Fuente, A., Martin-Pintado, J., Bachiller, R. et al. 1998, A\&A 334, 253

Harries, T.J., Hillier, D.J., Howarth, I.D. 1998, MNRAS 296, 1072

Hillier, D.J. 1996, A\&A 308, 521

Mannings, V., Koerner, D.W., Sargent, A.I. 1997, Nat. 388, 555

Oudmaijer, R.D., Proga, D., Drew, J.E., de Winter, D. 1998, MNRAS 300, 170

Oudmaijer, R.D. \& Drew, J.E. 1999, MNRAS 305, 166

Pezzuto, S., Strafella, F., Lorenzetti, D. 1997, ApJ 485, 290

Schulte-Ladbeck, R.E., Clayton, G.C., Hillier, D.J. et al. 1994, ApJ 429, 846

Thé, P.S., de Winter, D., Perez, M.R. 1994, A\&AS 104, 315

Zickgraf, F.-J. these proceedings 


\section{Discussion}

P. Najarro: How would the polarization look like if the winds of these stars were clumped? Could it mask the disk?

R. Oudmaijer: In a sense, the spectropolarimetry measures the geometry of the (electron) scattering material. If the clumps in the wind would be distributed nicely spherically symmetric, no line-effect would be found. If for some reason they would be distributed a-spherically, an effect should be visible, but it could be very weak as we measure the difference in numbers of scatterers in the different directions. In a disk-type case, you compare a scattering region (the disk) with no scattering at all (empty space perpendicular to the disk), while in a roughly spherically symmetric, but clumped wind, the differences may be much smaller. Ricardo Ignace presented a nice talk considering this point.

J. Marlborough: You mentioned HD 87643, is it a B[e] star or a Herbig star? R. Oudmaijer: It definitely is a B star with forbidden lines, and thus a B[e] star, but many Herbig Be stars show forbidden lines as well. Based on distance considerations (Oudmaijer et al. 1998), we found that it is more likely that HD 87643 is a luminous evolved $\mathrm{B}[\mathrm{e}]$ star located at several $\mathrm{kpc}$ rather than a less luminous 'Herbig B[e]' star located much closer to us. 